Journal for ImmunoTherapy of Cancer

\section{Phase II study of pembrolizumab and capecitabine for triple negative and hormone receptor-positive, HER2- negative endocrine-refractory metastatic breast cancer}

To cite: Shah AN, Flaum L, Helenowski I, et al. Phase II study of pembrolizumab and capecitabine for triple negative and hormone receptorpositive, HER2-negative endocrine-refractory metastatic breast cancer. Journal for ImmunoTherapy of Cancer 2020;8:e000173. doi:10.1136/ jitc-2019-000173

Accepted 16 January 2020
Check for updates

(C) Author(s) (or their employer(s)) 2020. Re-use permitted under CC BY-NC. No commercial re-use. See rights and permissions. Published by BMJ.

${ }^{1}$ Robert H Lurie Comprehensive Cancer Center of Northwestern University, Chicago, Illinois, USA ${ }^{2}$ Johns Hopkins Medicine Sidney Kimmel Comprehensive Cancer Center, Baltimore, Maryland, USA

Correspondence to

Dr Ami N Shah;

amishah@northwestern.edu

\section{ABSTRACT}

Background Response rates to single agent immune checkpoint blockade in unselected pretreated HER2negative metastatic breast cancer (MBC) are low. However, they may be augmented when combined with chemotherapy.

Methods We conducted a single-arm, phase II study of patients with triple negative (TN) or hormone receptorpositive endocrine-refractory ( $\mathrm{HR}+$ ) MBC who were candidates for capecitabine. Patients were treated with pembrolizumab $200 \mathrm{mg}$ intravenously day 1 and capecitabine $1000 \mathrm{mg} / \mathrm{m}^{2}$ by mouth twice daily on days 1-14 of a 21-day cycle. The primary end point was median progression-free survival (mPFS) compared with historic controls and secondary end points were overall response rate (ORR), safety and tolerability. The study had $80 \%$ power to detect a 2-month improvement in mPFS with the addition of pembrolizumab over historic controls treated with capecitabine alone.

Results Thirty patients, 16 TN and $14 \mathrm{HR}+\mathrm{MBC}$, were enrolled from 2017 to 2018. Patients had a median age of 51 years and received a median of 1 (range 0-6) prior lines of therapy for MBC. Of 29 evaluable patients, the mPFS was 4.0 ( $95 \% \mathrm{Cl} 2.0$ to 6.4$)$ months and was not significantly longer than historic controls of 3 months. The median overall survival was 15.4 (95\% Cl 8.2 to 20.3$)$ months. The ORR was $14 \%(n=4)$, stable disease (SD) was $41 \%(n=12)$ and clinical benefit rate $(\mathrm{CBR}=$ partial response+SD $>6$ months) was $28 \%$ $(\mathrm{n}=8)$. The ORR and CBR were not significantly different between disease subtypes (ORR 13\% and 14\%, CBR $25 \%$ and $29 \%$ for TN and HR+, respectively). The 1-year PFS rate was $20.7 \%$ and three patients have ongoing responses. The most common adverse events were low grade and consistent with those seen in MBC patients receiving capecitabine, including hand-foot syndrome, gastrointestinal symptoms, fatigue and cytopenias. Toxicities at least possibly from pembrolizumab included grade 3 or 4 liver test abnormalities (7\%), rash $(7 \%)$ and diarrhea (3\%), as well as grade 5 hepatic failure in a patient with liver metastases.
Conclusions Compared with historical controls, pembrolizumab with capecitabine did not improve PFS in this biomarker unselected, pretreated cohort. However, some patients had prolonged disease control. Trial registration number NCT03044730.

\section{BACKGROUND}

Patients with hormone receptor-positive (HR+) HER2-negative (HER2-) endocrinerefractory and triple negative (TN) metastatic breast cancer (MBC) are treated with sequential chemotherapy. ${ }^{1}{ }^{2}$ The median overall survival (mOS) with first-line chemotherapy in HER2- MBC is $<2$ years, indicating a large unmet need for novel therapeutic approaches. ${ }^{3} 4$ Capecitabine is often used as an early line of chemotherapy based on its efficacy, toxicity profile without significant cumulative toxicity and oral administration. ${ }^{5}$

Studies of pembrolizumab monotherapy, an antiprogrammed death (PD)-1 immune checkpoint inhibitor (ICI), have demonstrated improved overall response rates (ORR), progression-free survival (PFS) and OS with some patients having durable disease control in several malignancies. $^{67}$ ICI monotherapy in metastatic breast cancer (MBC) has generally had low response rates. $^{8-14}$ In previously treated TN MBC, ORR range from $5 \%$ to $10 \%$ in unselected patients, but reaches up to $25 \%$ in biomarker selected patients. In $\mathrm{HR}+$, HER2- endocrine-refractory MBC, that is, programmed death-ligand 1 (PD-L1)positive ORR was $12 \% .^{12}$ Some long-term responses have been seen in both cohorts. Response rates are higher in earlier lines of 
therapy, potentially related to less iatrogenic immunosuppression and lower tumor burden. ${ }^{13}{ }^{15}$

Combination chemo-immunotherapy has demonstrated safety and tolerability with improved ORR, PFS and OS in other malignancies. ${ }^{16}$ In a landmark study, the addition of anti-PD-L1 blockade with atezolizumab to chemotherapy (nab-paclitaxel) for first-line therapy in TN MBC resulted in improved PFS and OS in the PD-L1-positive cohort. ${ }^{15}$ Chemo-immunotherapy may have additive or even synergistic effects, with chemotherapy potentially producing neoantigens and disrupting mechanisms of tumor immune evasion (disrupting T-regulatory cell and myeloid-derived suppressor cell activity and promoting tumor cell recognition). ${ }^{17}$ Data from studies of capecitabine and antiPD-1 agents support the safety of the combination. ${ }^{18} 19$

The significant unmet need to improve outcomes, the low response rates to ICI monotherapy, the use of capecitabine as a standard early chemotherapy in MBC when patients are likely less immunosuppressed and the tolerability of chemo-immunotherapy in other trials provide the rationale for this phase II study of pembrolizumab and capecitabine in HR+ HER2- endocrinerefractory or TN MBC.

\section{PATIENTS AND METHODS \\ Patients}

Patients with HR+HER2- endocrine-refractory or TN MBC were included in this trial. Patients with HR+ HER2disease must have had progression on one or more lines of endocrine therapy. Patients were $\geq 18$ years, had an Eastern Cooperative Oncology Group performance status of 0-2 and adequate organ function. Those with active central nervous system disease, prior capecitabine, a history of autoimmune disease, active pneumonitis or prior severe pneumonitis requiring steroids or gastrointestinal disease that may impair capecitabine absorption were excluded.

\section{Trial design and procedures}

This was an open-label, single-arm, phase II study conducted at Northwestern University. After initial screening, patients were treated with pembrolizumab $200 \mathrm{mg}$ intravenously day 1 of a 21-day cycle and capecitabine $1000 \mathrm{mg} / \mathrm{m}^{2}$ by mouth twice daily days $1-14$ of a 21-day cycle. Toxicities were assessed after each cycle and response assessment by tumor imaging occurred every three cycles. Treatment continued until disease progression, unacceptable toxicity, withdrawal from the study, failure to adhere to recommendations or death. Treatment beyond radiographic progression was permitted with consent of patient in cases of no clinically significant change, no change in performance status or no rapid change or threat to vital organs.

The primary end point of PFS compared with historic controls and secondary end point of ORR were assessed by RECIST V.1.1 in the entire patient population and by subgroup (HR+ HER2- hormone-refractory and TN). A secondary end point of safety and tolerability was evaluated for all patients who received the study therapy by assessing adverse events using the Common Terminology Criteria for Adverse Events V.4.03. ORR and PFS by immune-RECIST (i-RECIST) was evaluated as an exploratory end point. Additionally, clinical benefit rate (CBR) defined as partial response (PR) and stable disease (SD) for $\geq 6$ months was assessed.

When tumor tissue was available from within 2 months prior to registration, a blinded external laboratory and pathologist assessed PD-L1 expression by immunohistochemistry (IHC) using the Merck 22C3 antibody, reported as a percentage $0 \%-100 \%$. PD-L1 positivity was defined as an IHC score $\geq 1+$ for $>1 \%$ of tumor cells. The modified proportion score of overall per cent cells expressing PD-L1 including lymphocytes and tumor cells, excluding staining of the surrounding stroma, was also evaluated. Tumor infiltrating lymphocytes (TILs) were assessed by H\&E stain and scored on a scale of $0-3$, with scores of 2 or 3 being considered high. Additionally, pathologists reported the presence of a stromal interface by a qualitative assessment of whether there was a distinct pattern of PD-L1 membrane stained cells within the stroma bordering tumors nests at low power and the majority of stained cells appear as macrophages at high power.

\section{Statistical approach}

The trial was designed to detect a clinically significant increase in PFS by 2 months from historic controls of 3 months in patients with prior therapy to 5 months. ${ }^{20-22}$ With 27 evaluable patients, there was an $80 \%$ power to detect this difference with a one-tailed z-test at $\mathrm{p}<0.05$. Categorical variables were compared between groups via Fisher's exact test and continuous variables were compared between groups via the Wilcoxon rank-sum test. Survival was compared using the log rank test.

\section{RESULTS}

From May 2017 to March 2018, 30 patients were enrolled at Northwestern University (table 1). One patient in the TN cohort was taken off study after one cycle because of failure to comply with capecitabine dosing recommendations and was not evaluable for the primary end point of PFS or for response. All patients were analyzed for safety and toxicity end points. The median age was 51 years. All patients with $\mathrm{HR}+$ endocrine-refractory and $88 \%$ of patients with TN MBC had visceral disease. Patients had a median of one line of prior systemic therapy for metastatic disease (range $0-6$ ), with a median of two lines (range 0-4) in the HR+ endocrine-refractory and one line (range 0-6) in the TN cohorts. This was the first line of systemic therapy for MBC for five TN and one HR+ patients, all of whom had received prior therapy for early stage disease. The study therapy was the second line of therapy for 11 patients, third line for 5 patients, fourth line for 5 patients and fifth or greater line for 3 patients. 
Table 1 Baseline characteristics

\begin{tabular}{|c|c|c|c|}
\hline & All $(n=30)$ & TN $(n=16)$ & $H R+(n=14)$ \\
\hline \multicolumn{4}{|l|}{ Clinical characteristics } \\
\hline Age, median (range) & $51(27-68)$ & $54.5(27-68)$ & $49(36-68)$ \\
\hline \multicolumn{4}{|l|}{ Race, \% (n) } \\
\hline White & $83 \%(25)$ & $88 \%(14)$ & $79 \%(11)$ \\
\hline Black & $10 \%(3)$ & $6 \%(1)$ & $14 \%(2)$ \\
\hline Unknown & $7 \%(2)$ & $6 \%(1)$ & $7 \%(1)$ \\
\hline \multicolumn{4}{|l|}{ Ethnicity, \% (n) } \\
\hline Non-Hispanic & $93 \%(28)$ & $94 \%(15)$ & $93 \%(13)$ \\
\hline Hispanic & $7 \%(2)$ & $6 \%(1)$ & $7 \%(1)$ \\
\hline \multicolumn{4}{|l|}{ ECOG PS, \% (n) } \\
\hline 0 & $57 \%(17)$ & $56 \%(9)$ & $57 \%(8)$ \\
\hline 1 & $40 \%(12)$ & $38 \%(6)$ & $43 \%(6)$ \\
\hline 2 & $3 \%(1)$ & $6 \%(1)$ & $0 \%(0)$ \\
\hline Visceral disease & $93 \%(28)$ & $88 \%(14)$ & $100 \%(14)$ \\
\hline \multicolumn{4}{|l|}{ Prior therapies for MBC } \\
\hline Total prior therapies, median (range) & $1(0-6)$ & $1(0-6)$ & $2(0-4)$ \\
\hline Endocrine therapy, median (range) & $0(0-4)$ & $\mathrm{N} / \mathrm{A}$ & $1(0-3)$ \\
\hline Chemotherapy, median (range) & $1(0-5)$ & $1(0-5)$ & $0(0-2)$ \\
\hline First line for MBC, \% (n) & $17 \%(7)$ & $31 \%(5)$ & $7 \%(1)$ \\
\hline
\end{tabular}

ECOG, Eastern Cooperative Oncology Group; HR+, hormone receptor-positive endocrine-refractory ; MBC, metastatic breast cancer ; N/A, not available; PS, performance status; TN, triple negative .

Patients received a median of five cycles of therapy. A dose reduction of capecitabine was required in $43 \%$ $(n=13)$ of patients, of whom three patients required two dose-level reductions. The most common reason for dose reduction was hand-foot syndrome. Pembrolizumab was interrupted for toxicity in $23 \%$ of patients. Therapy was stopped for progressive disease for $80 \% \quad(n=24)$, toxicity for $10 \%(\mathrm{n}=3)$, physician discretion $3 \%(\mathrm{n}=1$; for physician-determined clinical progression not meeting RECIST V.1.1 criteria for PD), and non-compliance 3\% $(n=1)$. One patient remains on trial.

Adverse events of any grade that occurred in at least onethird of patients that were at least possibly therapy-related were gastrointestinal (elevation in liver enzymes, nausea, diarrhea), fatigue, headache, hand-foot syndrome, pain in extremity, hematological (anemia, leukopenia, lymphopenia), hyperglycemia and hypoalbuminemia (table 2). Grade 3 or higher adverse events occurring in at least $10 \%$ of patients were an elevation in alkaline phosphatase, hand-foot syndrome, anemia and lymphopenia. One patient with $\mathrm{HR}+$ endocrine-refractory MBC, liver metastases at enrollment and preserved hepatic function had rapid disease progression and liver failure after two cycles. The patient received high-dose steroids and supportive care but died shortly after.

The median PFS (figure 1) was 4.0 (95\% CI 2.0 to 6.4) months, which did meet the prespecified threshold of a clinically meaningful 2-month increase in PFS and was not significantly longer than historic controls of 3 months. The median PFS was similar in the HR+ endocrine-refractory and TN cohorts, 5.1 (95\% CI 2.0 to 11.0 ) months and 4.0 (95\% CI 1.9 to 12.7 ) months, respectively, log-rank p value $=0.77$. Notably, $20.7 \%$ of patients were without progression at 1 year (95\% CI $8.4 \%$ to $36.7 \%$ ). The median OS was 15.4 (95\% CI 8.2, 20.3) months, with $63 \%$ of patients alive at 12 months $(95 \%$ CI $43.2 \%$ to $77.6 \%$ ). The median OS was not reached in the HR+ endocrine-refractory group and was 15.3 (95\% CI 4.4 to 19.4 ) months in the $\mathrm{TN}$ group.

The objective response rate (table 3, figure 2) was $14 \%$ and not significantly different between the TN and endocrine-refractory HR+ cohorts $(p=0.99)$. Similarly, the $\mathrm{CBR}$ ( $\mathrm{PR}+\mathrm{SD} \geq 6$ months) was not significantly different between subgroups and was $28 \%$ in the entire cohort. Evaluation of response by i-RECIST criteria showed no differences in response rates. There were no cases of pseudoprogression and no complete responses. Among the 17 patients for whom this was the first or second line of therapy for metastatic disease, no partial or complete responses were seen, although $18 \%$ derived clinical benefit with $>6$ months of SD.

Half of patients had evaluation of tissue from a metastatic site for expression of PD-L1 and immune infiltrate $(\mathrm{TN} n=5, \mathrm{HR}+\mathrm{n}=10$, table 4$)$. Tissue samples were obtained within 2 months of starting therapy and were from liver $(n=7,47 \%)$, lung $(n=2,13 \%)$, skin $(n=2,13 \%)$ 
Table 2 Treatment-related toxicities

\begin{tabular}{|c|c|c|c|}
\hline & Grade 1-2 & Grade $>3$ & All grades \\
\hline \multicolumn{4}{|l|}{ Gastrointestinal } \\
\hline Elevated akaline phosphatase & $57 \%$ & $10 \%$ & $67 \%$ \\
\hline Nausea & $53 \%$ & $0 \%$ & $53 \%$ \\
\hline Diarrhea & $47 \%$ & $3 \%$ & $50 \%$ \\
\hline Abdominal pain & $33 \%$ & $0 \%$ & $33 \%$ \\
\hline Constipation & $33 \%$ & $0 \%$ & $33 \%$ \\
\hline Vomiting & $30 \%$ & $0 \%$ & $30 \%$ \\
\hline Hepatic failure & $0 \%$ & $3 \%$ & $3 \%$ \\
\hline \multicolumn{4}{|l|}{ Dermatological and other } \\
\hline Headache & $40 \%$ & $0 \%$ & $40 \%$ \\
\hline Pain in extremity & $37 \%$ & $0 \%$ & $37 \%$ \\
\hline Back pain & $33 \%$ & $0 \%$ & $33 \%$ \\
\hline Sinus tachycardia & $30 \%$ & $0 \%$ & $30 \%$ \\
\hline Hypertension & $20 \%$ & $7 \%$ & $27 \%$ \\
\hline Edema & $27 \%$ & $0 \%$ & $27 \%$ \\
\hline Maculopapular rash & $13 \%$ & $3 \%$ & $17 \%$ \\
\hline Peripheral neuropathy & $17 \%$ & $0 \%$ & $17 \%$ \\
\hline \multicolumn{4}{|l|}{ Hematological } \\
\hline Hyperglycemia & $83 \%$ & $3 \%$ & $87 \%$ \\
\hline Hypoalbuminemia & $33 \%$ & $3 \%$ & $37 \%$ \\
\hline Hypokalemia & $30 \%$ & $3 \%$ & $33 \%$ \\
\hline Acute kidney injury & $0 \%$ & $3 \%$ & $3 \%$ \\
\hline
\end{tabular}

ALT, alanine transaminase; AST, aspartate transaminase.

and adrenal, bladder and breast ( $\mathrm{n}=1$ for each category, $7 \%$ ). Sixty per cent of samples were PD-L1 negative (IHC score 0 for tumor cells), $70 \%$ and $40 \%$ were PD-L1 negative in $\mathrm{HR}+$ endocrine-refractory and $\mathrm{TN}$ cohorts, respectively. TIL scores were $7 \%, 47 \%, 27 \%, 20 \%$ for scores 0,1 , 2 and 3, respectively. PD-L1 positivity or high TILs did not correlate with response to therapy.

The median change in lymphocyte count from day 1 of treatment to day 1 of the last cycle on study was a decrease by 0.1 lymphocyte $/ \mu \mathrm{L}$. Lymphopenia was present at baseline in $52 \%$ of patients and at end of treatment in $67 \%$ of patients. There was no statistically significant difference in rate of lymphopenia in responders compared with non-responders (table 5).

Eight patients had clinical benefit (four PR, four SD $\geq 6$ months) of whom six patients remained on therapy without progression for longer than 1 year (three TN, three $\mathrm{HR}+$ endocrine-refractory disease). However, one HR+ patient had only $9 \%$ expression of the estrogen receptor and $3 \%$ of the progesterone receptor. Among these patients with clinical benefit, the median lines of prior chemotherapy for metastatic disease was 1 (range 0-2). Three had a correlative tissue sample (one TN, two $\mathrm{HR}+)$. These patients had a PD-L1 tumor proportion 


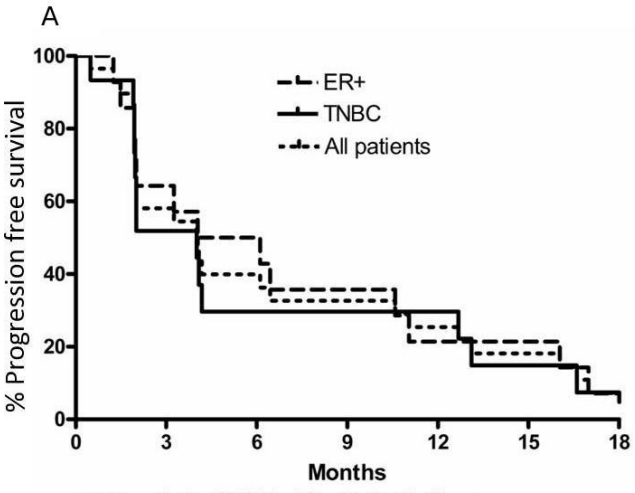

$\mathrm{mPFS}=4.0(95 \% \mathrm{CI}, 2.0-6.4) \mathrm{mo}$

TN mPFS $=4.0(95 \% \mathrm{Cl}, 1.9-12.7) \mathrm{mo}$

$\mathrm{HR}+\mathrm{mPFS}=5.1(95 \% \mathrm{Cl}, 2.0-11.0) \mathrm{mo}$

12 -mo PFS rate $=20.7 \%(95 \% \mathrm{Cl},(8.4 \%, 36.7 \%)$

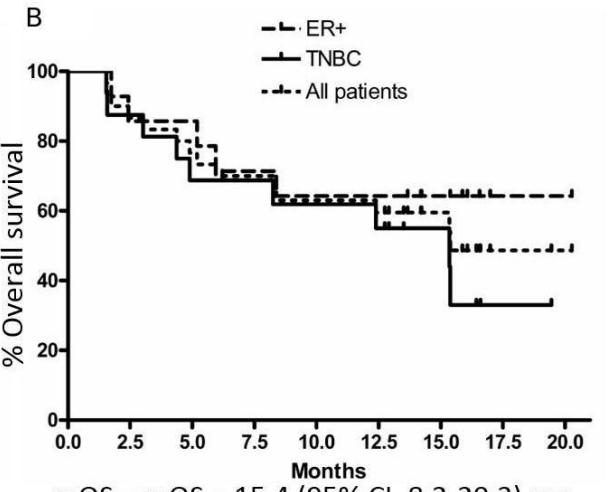

$\mathrm{mOS}=\mathrm{mOS}=15.4(95 \% \mathrm{Cl}, 8.2-20.3) \mathrm{mo}$

TN: $\mathrm{mOS}=15.3(95 \% \mathrm{Cl} 4.4-19.4) \mathrm{mo}$

$\mathrm{HR}+\mathrm{mOS}=$ not reached

$12-$ mo OS rate $=63 \%(43.2 \%, 77.6 \%)$

Figure 1 (A) Progression-free (PFS) and (B) overall survival (OS) in all patients and by breast cancer subtype. ER+, endocrinerefractory-positive; HR+, hormonereceptor-positive endocrine-refractory; TNBC, triple negative breast cancer.

score (\% of tumor cells with PD-L1 IHC >1+) of $0 \%, 0 \%$ and $3 \%$, and TIL scores (scale $0-3$ ) of 2,1 and 1 (table 4 ).

\section{DISCUSSION}

Pembrolizumab with capecitabine did not improve PFS compared with historic controls in this biomarker unselected cohort that consisted of patients with prior systemic therapy. This finding is consistent with the limited efficacy of anti-PD-(L) 1 monotherapy in unselected, pretreated MBC. ${ }^{911}$ Despite the lack of selection, $20 \%$ of patients had disease control beyond 1year of therapy and 28\% derived clinical benefit with the combination therapy.

In addition to subtype and prior therapies, PD-L1 and TILs for those with available tissue and lymphocyte count were considered in an exploratory analysis as potential biomarkers for enhanced response to therapy. Prior studies have demonstrated PD-L1 on TILs can predict for immunotherapy benefit in metastatic TNBC and studies in PD-L1-positive patients with breast cancer have higher response rates than in unselected patients. ${ }^{815}$ High TILs have also been associated with better clinical outcomes in metastatic TNBC. ${ }^{23}$ Lymphocyte count was explored as it

\begin{tabular}{lcll}
\hline Table 3 Response rate & & & \\
\hline & All & TN (n=15) & $\begin{array}{l}\text { HR+ } \\
(\mathbf{n = 1 4 )}\end{array}$ \\
\hline Objective response rate & $4(14 \%)$ & $2(13 \%)$ & $2(14 \%)$ \\
\hline Partial response (PR) & $4(14 \%)$ & $2(13 \%)$ & $2(14 \%)$ \\
\hline Stable disease (SD) & $12(41 \%)$ & $5(33 \%)$ & $7(50 \%)$ \\
\hline Progressive disease & $13(43 \%)$ & $8(50 \%)$ & $5(36 \%)$ \\
Not evaluable & $1(3 \%)$ & $1(6 \%)$ & 0 \\
\hline $\begin{array}{l}\text { Clinical benefit rate } \\
\text { (=PR+SD >6 months) }\end{array}$ & $8(28 \%)$ & $4(27 \%)$ & $4(28 \%)$ \\
\hline
\end{tabular}

$\mathrm{HR}+$, hormone receptor-positive endocrine-refractory ; TN, triple negative. may be a surrogate for immune response and may reflect degree of immunosuppression from prior therapy. ${ }^{24} 25$

No factor clearly correlated with clinical benefit including subtype (HR+ HER2- endocrine-refractory vs $\mathrm{TN}$ ), lines of prior therapy for metastatic disease, PD-L1 status, TIL level or lymphocyte count; however, this subgroup analysis was limited by a modest patient cohort size. Future studies with larger patient cohorts and randomized designs should continue to explore potential predictors of response to identify the smaller subset of patients who may benefit from a combined chemotherapy and immunotherapy approach.

Several studies have demonstrated promising findings for chemo-immunotherapy in TNBC. Most notably, the IMpassion130 study demonstrated atezolizumab and

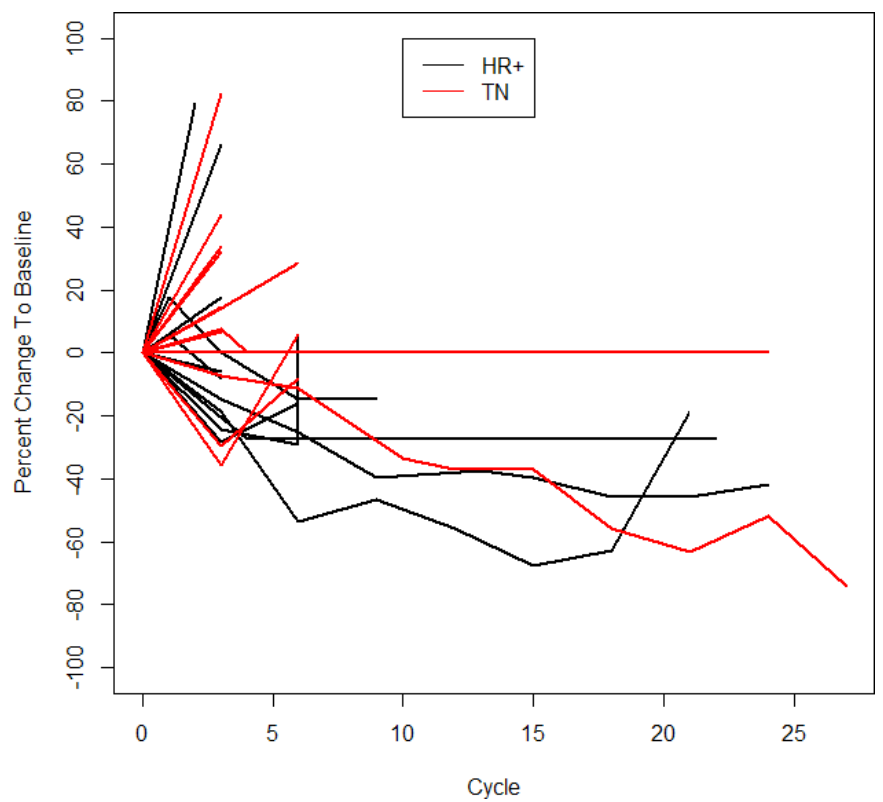

Figure 2 Response to capecitabine and pembrolizumab by RECIST V.1.1 criteria. HR+, hormonereceptor-positive endocrine-refractory; TN, triple negative. 
Table 4 Tissue immune correlatives

\begin{tabular}{|c|c|c|c|c|c|c|c|}
\hline Subgroup & Tissue site & TPS & $\begin{array}{l}\text { PD-L1 H- } \\
\text { score }\end{array}$ & MPS & $\begin{array}{l}\text { TIL score } \\
(0-3)\end{array}$ & $\begin{array}{l}\text { Stromal } \\
\text { interface }\end{array}$ & $\begin{array}{l}\text { Best } \\
\text { response }\end{array}$ \\
\hline \multicolumn{8}{|c|}{ Clinical benefit (CR, $P R$ or $S D \geq 6$ months) } \\
\hline $\mathrm{HR}+$ & Liver & 97 & 3 & 3 & 1 & No & PR \\
\hline $\mathrm{HR}+$ & Adrenal & 100 & 0 & 0 & 1 & No & PR \\
\hline
\end{tabular}

No clinical benefit (PD or $S D<6$ months)

\begin{tabular}{llllllll} 
HR+ & Lymph node & 100 & 0 & 0 & 3 & Yes & PD \\
HR+ & Skin & 100 & 0 & 0 & 0 & No & PD \\
HR+ & Lung & 100 & 0 & 0 & 2 & No & SD \\
HR+ & Liver & 100 & 0 & 0 & 1 & No & SD \\
HR+ & Liver & 100 & 0 & 0 & 1 & No & SD \\
HR+ & Liver & 100 & 0 & 0 & 1 & Yes & PD \\
HR+ & Liver & 98 & 2 & 2 & 2 & No & PD \\
TN & Liver & 95 & 11 & 5 & 3 & Yes & SD \\
TN & Lung & 100 & 0 & 0 & 1 & Yes & PD \\
TN & Liver & 10 & 210 & 90 & 3 & No & PD \\
TN & Skin & 98 & 2 & 2 & 1 & No & PD \\
\hline
\end{tabular}

TPS $=\%$ of PD-L1-positive $(\mathrm{IHC}>1+)$ tumor cells; PD-L1 H-score $=(\%$ IHC $1+\times 1)+(\%$ IHC $2+\times 2)+(\%$ IHC $3+\times 3)$; MPS-TPS with mononuclear inflammatory cells expressing PD-L1 also included.

.CR, complete response; HR+, hormone receptor-positive endocrine-refractory; IHC, immunohistochemistry; MPS, modified proportion score; PD, progressive disease ; PD-L1, programmed death-ligand 1 ; PR, partial response ; SD, stable disease ; TN, triple negative; TPS, tumor proportion score.

Table 5 Immune correlatives and clinical benefit rate based on (A) metastatic tissue sample and (B) absolute lymphocyte count

\begin{tabular}{|c|c|c|}
\hline (A) & $\begin{array}{l}\text { PD-L1 positive } \\
(n=6)\end{array}$ & $\begin{array}{l}\text { PD-L1 negative } \\
(n=9)\end{array}$ \\
\hline \multirow{2}{*}{$\begin{array}{l}\text { Clinical benefit } \\
\text { rate }\end{array}$} & $33.3 \%(n=2)$ & $22.2 \%(n=2)$ \\
\hline & TIL high $(2+$ or $3+)$ & TIL low (0 or $1+$ ) \\
\hline $\begin{array}{l}\text { Clinical benefit } \\
\text { rate }\end{array}$ & $28.6 \%(n=2)$ & $25 \%(n=2)$ \\
\hline (B) & $\begin{array}{l}\text { Clinical benefit } \\
(n=8)\end{array}$ & $\begin{array}{l}\text { No clinical benefit } \\
(n=21)\end{array}$ \\
\hline $\begin{array}{l}\text { Lymphopenia } \\
\text { C1D1 }\end{array}$ & $25 \%(n=2)$ & $42.9 \%(n=9$ of 21$)$ \\
\hline $\begin{array}{l}\text { Lymphopenia } \\
\text { C3D1 }\end{array}$ & $50 \%(n=4)$ & $\begin{array}{l}33.3 \%(n=6 \text { of } 18 \\
\text { with data) }\end{array}$ \\
\hline $\begin{array}{l}\text { Lymphopenia end } \\
\text { of treatment }\end{array}$ & $50 \%(n=4)$ & $47.6 \%(n=10$ of 21$)$ \\
\hline
\end{tabular}

PD-L1, programmed death-ligand 1 ; TIL, tumor infiltrating lymphocyte. nab-paclitaxel improved OS compared with chemotherapy alone when given as a first-line therapy in a cohort with PD-L1-positive lymphocytes $>1 \%$ and TN MBC. ${ }^{15}$ This combination of capecitabine with pembrolizumab in TNBC was evaluated in a recently reported phase II study that included 14 patients in the capecitabine arm and demonstrated a 12-week ORR of $43 \%$ and mPFS of 5.6 months. The higher response rate may be related to its use in earlier lines of therapy (first-line for $79 \%$ of patients). Of note, the ORR, CBR, PFS and OS numerically favored the capecitabine-pembrolizumab cohort over the paclitaxel-pembrolizumab group. ${ }^{26}$ Additional data from early stage breast cancer studies also demonstrate the promise of chemo-immunotherapy in TNBC. The I-SPY 2 phase II trial of neoadjuvant chemotherapy with paclitaxel and pembrolizumab followed by doxorubicin and cyclophosphamide showed improvement in the estimated pathologic complete response (pCR) rate from $22.3 \%$ to $62.4 \%$ in the TN cohort. ${ }^{27}$ The KEYNOTE-522 study was a large randomized phase III trial that demonstrated the addition of neoadjuvant and adjuvant pembrolizumab to a chemotherapy regimen of a taxane+carboplatin followed by an anthracycline+cyclophosphamide with 
adjuvant pembrolizumab improved the pCR rate from $51.2 \%$ to $64.8 \% .^{28}$ Results from the phase III IMpassion132 trial evaluating atezolizomib with several chemotherapies including capecitabine as first-line therapy for TN MBC will contribute to our understanding of the use of chemo-immunotherapy in metastatic TNBC. ${ }^{29}$

For HR+ endocrine-refractory $\mathrm{MBC}$, there is more limited data for chemo-immunotherapy. One phase II study of eribulin and pembrolizumab compared with pembrolizumab alone demonstrated no improvement in PFS. ${ }^{30}$ The I-SPY 2 phase II trial showed improvement in the estimated pCR rate among patients with previously untreated HR+ HER2- early stage breast cancer from $13.6 \%$ to $34.2 \% .{ }^{27}$ Additionally, several ongoing trials are evaluating chemo-immunotherapy in HER2- MBC (eg, NCT02752685, UMIN000030242, NCT03371017).

Most toxicities were low grade and consistent with those expected with capecitabine monotherapy in MBC. This included elevation of liver tests, cytopenias (including lymphopenia of any grade occurring in 53\% of patients), skin rash and fatigue. However, higher grade toxicities were also observed. There was one death from hepatic failure and rapid disease progression. It remains unclear whether this death was a result of rapid disease progression as can be seen in patients with MBC and liver metastases, immune-related hepatitis, a change in the character and pace of disease related to immunotherapy or a combination of these potential causes. Such an acceleration in the disease with ICI has been described in other malignancies as hyperprogressive disease, although the exact definition and relationship with immunotherapy has not been established. ${ }^{31-33}$ Future studies of immunotherapy in MBC with liver metastases should include careful monitoring of liver function and may provide added insight about whether hyperprogression is a true phenomenon observed in breast cancer.

Despite the lack of improvement in mPFS, the tolerability of the combination and noting a subset of patients with clinical benefit in the context of growing data supporting chemo-immunotherapy in MBC warrants further exploration of the combination in a more selected population (earlier line of therapy with more rigorous evaluation for predictive biomarkers). Future studies of chemo-immunotherapy in MBC are needed to identify clinical, pathological and molecular predictors of response and toxicity to identify subgroups more likely to benefit from the addition of ICI to chemotherapy.

\section{CONCLUSIONS}

Compared with historical controls, pembrolizumab with capecitabine did not demonstrate a statistically significant improvement in PFS in a biomarker unselected, pretreated cohort. However, some patients had prolonged disease control, including patients with HR+ HER2 MBC. Future studies of chemo-immunotherapy should select for factors likely to augment benefit, such as earlier lines of therapy and predictive biomarkers.
Contributors Conception and design: SJ, AR and WG. Provision of patients: LF, WG, MC, VN, DT and CAS-M. Collection and assembly of data, data analysis and interpretation: all authors. Manuscript writing: ANS and IH. Manuscript approval: all authors.

Funding This study was funded by Merck.

Competing interests No, there are no competing interests.

\section{Patient consent for publication Not required.}

Ethics approval The study was reviewed and approved by the Northwestern University IRB, STU00203215. All procedures were conducted in accordance with the Good Clinical Practice and the Declaration of Helsinki. All patients signed consent prior to any study-related procedures.

Provenance and peer review Not commissioned; externally peer reviewed.

Data availability statement Data are available on reasonable request.

Open access This is an open access article distributed in accordance with the Creative Commons Attribution Non Commercial (CC BY-NC 4.0) license, which permits others to distribute, remix, adapt, build upon this work non-commercially, and license their derivative works on different terms, provided the original work is properly cited, appropriate credit is given, any changes made indicated, and the use is non-commercial. See http://creativecommons.org/licenses/by-nc/4.0/.

\section{ORCID iD}

Ami N Shah http://orcid.org/0000-0003-1347-3782

\section{REFERENCES}

1 Telli ML, Gradishar WJ, Ward JH. NCCN guidelines updates: breast cancer. J Nat/ Compr Canc Netw 2019;17:552-5.

2 Partridge $\mathrm{AH}$, Rumble RB, Carey LA, et al. Chemotherapy and targeted therapy for women with human epidermal growth factor receptor 2-negative (or unknown) advanced breast cancer: American Society of clinical oncology clinical practice guideline. J Clin Oncol 2014;32:3307-29.

3 Stockler MR, Harvey VJ, Francis PA, et al. Capecitabine versus classical cyclophosphamide, methotrexate, and fluorouracil as first-line chemotherapy for advanced breast cancer. JCO 2011;29:4498-504

4 Rugo HS, Barry WT, Moreno-Aspitia A, et al. Randomized phase III trial of paclitaxel once per week compared with nanoparticle albumin-bound nab-paclitaxel once per week or ixabepilone with bevacizumab as first-line chemotherapy for locally recurrent or metastatic breast cancer: CALGB 40502/NCCTG N063H (Alliance). JCO 2015;33:2361-9.

5 O'Shaughnessy JA, Kaufmann M, Siedentopf F, et al. Capecitabine monotherapy: review of studies in first-line HER-2-negative metastatic breast cancer. Oncologist 2012;17:476-84.

6 Reck M, Rodríguez-Abreu D, Robinson AG, et al. Pembrolizumab versus chemotherapy for PD-L1-positive non-small-cell lung cancer. N Engl J Med 2016;375:1823-33.

7 Robert C, Schachter J, Long GV, et al. Pembrolizumab versus ipilimumab in advanced melanoma. New England Journal of Medicine 2015;372:2521-32.

8 Adams S, Schmid P, Rugo HS, et al. Pembrolizumab monotherapy for previously treated metastatic triple-negative breast cancer: cohort a of the phase II KEYNOTE-086 study. Ann Oncol 2019;30:397-404.

9 Nanda R, Chow LQM, Dees EC, et al. Pembrolizumab in patients with advanced triple-negative breast cancer: phase Ib KEYNOTE-012 study. JCO 2016;34:2460-7.

10 Dirix LY, Takacs I, Jerusalem G, et al. Avelumab, an anti-PD-L1 antibody, in patients with locally advanced or metastatic breast cancer: a phase 1B javelin solid tumor study. Breast Cancer Res Treat 2018;167:671-86.

11 Emens LA, Cruz C, Eder JP, et al. Long-Term clinical outcomes and biomarker analyses of Atezolizumab therapy for patients with metastatic triple-negative breast cancer. JAMA Oncol 2019;5:74-82.

12 Rugo HS, Delord J-P, Im S-A, et al. Safety and antitumor activity of pembrolizumab in patients with estrogen Receptor-Positive/Human epidermal growth factor receptor 2-Negative advanced breast cancer. Clin Cancer Res 2018;24:2804-11.

13 Adams S, Loi S, Toppmeyer D, et al. Pembrolizumab monotherapy for previously untreated, PD-L1-positive, metastatic triple-negative breast cancer: cohort B of the phase II KEYNOTE-086 study. Ann Oncol 2019;30:405-11.

14 Adams S. Current landscape of immunotherapy in breast cancer: a ReviewCurrent landscape of immunotherapy in breast CancerCurrent 
landscape of immunotherapy in breast cancer. JAMA Oncology 2019;5:1205-14.

15 Schmid P, Adams S, Rugo HS, et al. Atezolizumab and nabpaclitaxel in advanced triple-negative breast cancer. N Engl J Med 2018;379:2108-21.

16 Gandhi L, Rodríguez-Abreu D, Gadgeel S, et al. Pembrolizumab plus chemotherapy in metastatic non-small-cell lung cancer. $N$ Engl $J$ Med 2018;378:2078-92.

17 Emens LA, Middleton G. The interplay of immunotherapy and chemotherapy: harnessing potential synergies. Cancer Immunol Res 2015;3:436-43.

18 Page DB, Kim IK, Sanchez K, et al. Safety and efficacy of pembrolizumab (pembro) plus capecitabine (CAPE) in metastatic triple negative breast cancer (mTNBC). Journal of Clinical Oncology 2018;36:1033.

19 Boku N, Ryu M-H, Kato K, et al. Safety and efficacy of nivolumab in combination with S-1/capecitabine plus oxaliplatin in patients with previously untreated, unresectable, advanced, or recurrent gastric/ gastroesophageal junction cancer: interim results of a randomized, phase II trial (ATTRACTION-4). Annals of Oncology 2019;30:250-8.

20 Rugo HS, Roche H, Thomas E, et al. Efficacy and safety of ixabepilone and capecitabine in patients with advanced triplenegative breast cancer: a pooled analysis from two large phase III, randomized clinical trials. Clin Breast Cancer 2018;18:489-97.

21 Kaufman PA, Awada A, Twelves C, et al. Phase III open-label randomized study of eribulin mesylate versus capecitabine in patients with locally advanced or metastatic breast cancer previously treated with an anthracycline and a taxane. JCO 2015;33:594-601.

22 Zhang P, Sun T, Zhang Q, et al. Utidelone plus capecitabine versus capecitabine alone for heavily pretreated metastatic breast cancer refractory to anthracyclines and taxanes: a multicentre, open-label, superiority, phase 3, randomised controlled trial. Lancet Oncol 2017;18:371-83.

23 Loi S, Adams S, Schmid P, et al. LBA13Relationship between tumor infiltrating lymphocyte (TIL) levels and response to pembrolizumab (pembro) in metastatic triple-negative breast cancer (mTNBC): results from KEYNOTE-086. Annals of Oncology 2017;28.
24 Gibney GT, Weiner LM, Atkins MB. Predictive biomarkers for checkpoint inhibitor-based immunotherapy. Lancet Oncol 2016;17:e542-51.

25 Diehl A, Yarchoan M, Hopkins A, et al. Relationships between lymphocyte counts and treatment-related toxicities and clinical responses in patients with solid tumors treated with PD-1 checkpoint inhibitors. Oncotarget 2017;8:114268-80.

26 Page DB, Chun B, Pucilowska J, et al. Pembrolizumab (pembro) with paclitaxel (Taxol) or capecitabine (CAPE) as early treatment of metastatic triple-negative breast cancer (mTNBC). Journal of Clinical Oncology 2019;37:1015

27 Nanda R, Liu MC, Yau C, et al. Pembrolizumab plus standard neoadjuvant therapy for high-risk breast cancer (bc): results from I-SPY 2. Journal of Clinical Oncology 2017;35:506.

28 Schmid P, Cortés J, Dent R, et al. KEYNOTE-522: phase III study of pembrolizumab (pembro) + chemotherapy (chemo) vs placebo (pbo) + chemo as neoadjuvant treatment, followed by pembro vs pbo as adjuvant treatment for early triple-negative breast cancer (TNBC). Annals of Oncology 2019;30:v853-4.

29 Cortés J, André F, Gonçalves A, et al. IMpassion132 phase III trial: atezolizumab and chemotherapy in early relapsing metastatic triplenegative breast cancer. Future Oncology 2019;15:1951-61.

30 Tolaney SM, Barroso-Sousa R, Keenan T, et al. Randomized phase II study of eribulin mesylate $(E)$ with or without pembrolizumab $(P)$ for hormone receptor-positive (HR+) metastatic breast cancer (MBC). Journal of Clinical Oncology 2019;37:1004.

31 Ferrara R, Mezquita L, Texier M, et al. Hyperprogressive disease in patients with advanced Non-Small cell lung cancer treated with PD-1/PD-L1 inhibitors or with single-agent chemotherapy. JAMA Oncol 2018;4:1543-52.

32 Champiat S, Dercle L, Ammari S, et al. Hyperprogressive disease is a new pattern of progression in cancer patients treated by anti-PD-1/ PD-L1. Clin Cancer Res 2017;23:1920-8.

33 Champiat S, Ferrara R, Massard C, et al. Hyperprogressive disease: recognizing a novel pattern to improve patient management. Nat Rev Clin Oncol 2018;15:748-62. 